\title{
EchoGéo
}

$30 \mid 2014$

Varia

\section{La gestion des déchets post-catastrophe à Port-au- Prince : entre relégation et proximité}

Roxana Popescu, Mathieu Durand and Robert d'Ercole

\section{(2) OpenEdition \\ 12 Journals}

Electronic version

URL: https://journals.openedition.org/echogeo/14070

DOI: 10.4000/echogeo.14070

ISSN: 1963-1197

Publisher

Pôle de recherche pour l'organisation et la diffusion de l'information géographique (CNRS UMR 8586)

\section{Electronic reference}

Roxana Popescu, Mathieu Durand and Robert d'Ercole, "La gestion des déchets post-catastrophe à Port-au-Prince : entre relégation et proximité", EchoGéo [Online], 30 | 2014, Online since 11 December 2014, connection on 31 July 2021. URL: http://journals.openedition.org/echogeo/14070 ; DOI: https:// doi.org/10.4000/echogeo.14070

This text was automatically generated on 31 July 2021.

EchoGéo est mis à disposition selon les termes de la licence Creative Commons Attribution - Pas d'Utilisation Commerciale - Pas de Modification 4.0 International (CC BY-NC-ND) 


\title{
La gestion des déchets post- catastrophe à Port-au-Prince : entre relégation et proximité
}

\author{
Roxana Popescu, Mathieu Durand and Robert d'Ercole
}

1 La ville de Port-au-Prince, capitale haïtienne, connaît régulièrement des catastrophes engendrant chaque fois de grandes quantités de déchets, comme ce fut le cas suite au séisme de 2010. Aux déchets produits par ces très grandes catastrophes s'ajoutent ceux de phénomènes de moindre ampleur, mais plus réguliers (ouragans, inondations, mouvements de terrain). Ces déchets représentent donc de grandes quantités, sans que leur gestion n'ait jamais fait l'objet d'une grande attention, de la part des spécialistes des déchets comme de ceux des situations de crise. En cas de catastrophe, ce secteur semble en effet moins prioritaire que la recherche des survivants ou la fourniture en ressources vitales (eau, électricité, logement, soins d'urgence, etc.). La mise en place de sanitaires dans les camps de réfugiés, destinés au traitement des rejets de type "déchets liquides », est une priorité afin d'éviter les épidémies telles que le choléra (COOPI, IRD, 2011). Ce n'est pas encore le cas des déchets solides et des débris d'une catastrophe.

2 Pourtant, au-delà du risque sanitaire lié à leur présence, les décombres compromettent l'arrivée des secours et les ravitaillements, venant obstruer les voies de communication. Ils sont également source d'inondation en bouchant les canaux d'irrigation et les ravines. À plus long terme, les retours d'expériences internationaux montrent également qu'une mauvaise gestion des déchets post-catastrophe handicape la remise en état des services de base, la reconstruction et le redressement économique du territoire affecté. Tous les bilans réalisés suite à la gestion des situations de crise insistent sur la nécessité de gérer au mieux ces déchets, de les valoriser autant que possible, en s'appuyant sur les ressources humaines et matérielles locales, ainsi que sur la nécessité d'anticiper cette gestion. Ce sont par exemple les conclusions tirées de la gestion du séisme de Marmara en Turquie en 1999, du tsunami d'Asie du Sud-Est en 2004 ou de l'ouragan Katrina en Louisiane en 2005 (Brown et al., 2011). 
3 C'est dans ce contexte que nous avons cherché à mieux comprendre la gestion des déchets du séisme de 2010 à Port-au-Prince en Haïti. L'analyse s'appuie sur l'étude ${ }^{1}$ de la situation des déchets en situation normale dans la commune de Tabarre ${ }^{2}$ (une des communes de l'agglomération urbaine de Port-au-Prince), ainsi que sur la gestion effectuée des déchets post-catastrophe depuis 2010. Ce texte porte plus particulièrement sur l'analyse des logiques spatiales ${ }^{3}$ de gestion des déchets postcatastrophe à Port-au-Prince, et notamment à Tabarre. Nous observerons deux logiques qui se complètent, malgré une apparente contradiction : la relégation et la proximité. Il s'agit de phénomènes couramment observés dans la gestion des déchets quotidiens mais qui sont exacerbés en situation de crise. Avant de préciser les enjeux de cette analyse spatiale, nous reviendrons sur l'importance de la prise en compte de la question des déchets post-catastrophe.

\section{Impacts multiscalaires des déchets post-catastrophe}

\section{Une définition fluctuante des déchets post-catastrophe}

4 Avant de comprendre la spécificité de la gestion des déchets en situation de crise, et ce à travers plusieurs échelles d'analyse, il convient de revenir sur cet objet dont la définition n'est pas encore tout à fait stabilisée. Les déchets post-catastrophe incluent les débris issus des destructions et des démolitions de bâtiments, ainsi que les déchets ménagers que la population continue de produire, souvent dans des conditions inhabituelles (par exemple dans les camps de déplacés). Les institutions internationales qui, en cas de défaillance des États, prennent en charge la gestion de crise (ce fut le cas à Haïti suite au séisme de 2010), utilisent le terme de "débris " pour caractériser les déchets produits par une catastrophe. C'est d'ailleurs le mot qui a été utilisé pour les plans «débris 1 » et "débris 2 ", lancés par le PNUD, qui ont permis de gérer les déchets produits à Port-au-Prince (PNUD, 2013). Ce terme a également été repris par l'ensemble des institutions publiques haïtiennes et des ONG internationales, même si dans le langage courant il est souvent interverti avec celui de "décombres» ou de "gravats».

5 Ce terme de débris fait référence d'une part à l'origine des déchets, c'est-à-dire les décombres de bâtiments effondrés, d'autre part à leur nature, celle de gravats (déchets inertes). Cette double étymologie revêt une connotation négative, puisqu'elle est associée aux produits de la destruction de la ville. De plus, le terme de débris tend à restreindre la diversité des types de déchets pris en compte, puisqu'il n'inclut que les déchets inertes, assimilables à des déchets du bâtiment et des travaux publics. Il écarte ainsi les déchets dangereux, les déchets organiques et les déchets valorisables.

6 Nous préférons alors utiliser le terme de déchets post-catastrophe, de plus en plus employé dans la littérature scientifique (Robin des bois, 2007 ; Beraud et al., 2012), connoté de façon plus positive. La typologie peut alors s'élargir, incluant des décombres de bâtiments, des véhicules hors d'usage, des cadavres humains ou animaux, des déchets dangereux ou des déchets ménagers que la population continue de produire. Cette terminologie de déchets post-catastrophe met en relief la nature très diverse des déchets et surtout les possibilités de valorisations différenciées. 


\section{Les risques liés à la présence de déchets ménagers et de déchets de la construction}

7 La présence non contrôlée de déchets induit un certain nombre de nuisances potentielles. Il faut alors prendre en compte le risque sanitaire classiquement associé aux déchets (Ngo et Régent, 2008), en tant que vecteur de pathologie et de contamination des ressources naturelles. Par ailleurs, les déchets post-catastrophe étant en grande partie constitués de déchets inertes (similaires à des déchets de la construction), le risque sanitaire est moins important que pour les déchets ménagers. Ce risque sanitaire limité est circonscrit aux déchets organiques ou dangereux. Ce constat ne diminue pas pour autant les risques liés aux déchets post-catastrophe. Ils peuvent en effet prendre une autre ampleur lorsqu'ils viennent obstruer des cours d'eau, des canalisations, et ainsi engendrer une modification massive des flux hydriques. Les inondations et les éboulements de terrain sont ainsi les premiers risques liés aux déchets pris en compte lors de catastrophe.

Illustration 1 - Encombrement des ravines de Tabarre par des déchets

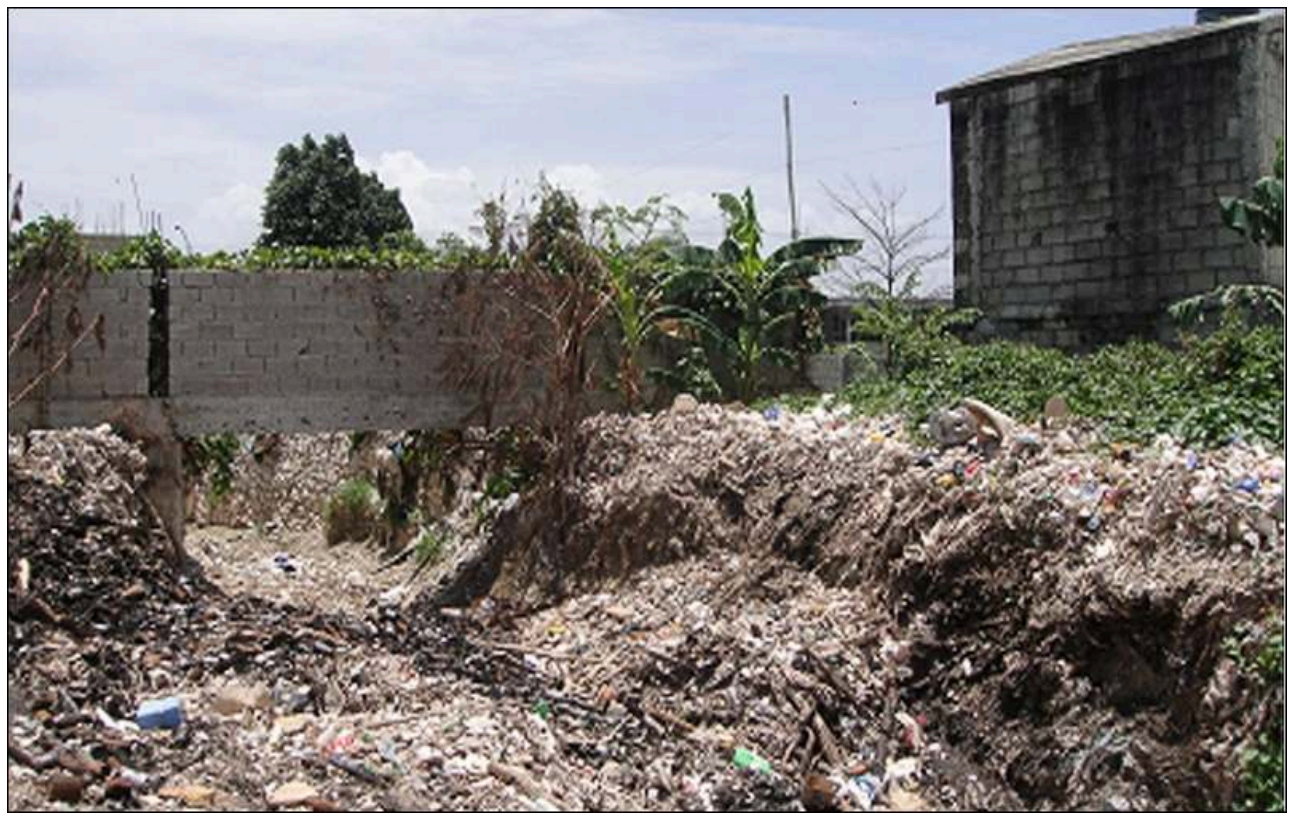

Auteur : R. Popescu, 2011

Dans le cas de Port-au-Prince, la prolifération de déchets présente un risque particulier en raison de la topographie. La ville est encastrée entre des reliefs pentus ${ }^{4}$. La pluviométrie y est importante et brutale, avec une valeur maximale de $315 \mathrm{~mm}$ pendant 24 heures (Bozza, 2012). Un grand nombre de vallées sèches et de canaux permet alors d'évacuer les eaux vers les communes de la plaine, dont la plus exposée est la commune de Tabarre (illustration 2). Les déchets, qu'ils soient produits en dehors ou pendant les situations de crise $^{5}$, viennent alors obstruer ces exutoires, provoquant localement des inondations parfois sévères (COOPI, IRD, 2011). Les responsables du Ministère des Travaux Publics, des Transports et de la Communication (MTPTC) admettent d'ailleurs ${ }^{6}$ que la question des déchets produits par le séisme de 2010 a commencé à être traitée uniquement lorsque les inondations menaçaient. Ce fut le cas trois mois après la catastrophe, avec l'arrivée de la saison des pluies. 
La commune de Tabarre, sur laquelle s'est opéré ce travail de recherche a une situation particulière dans la ville. Elle était, avant le séisme, une commune périphérique avec beaucoup de zones agricoles disponibles. La présence sur son territoire de l'aéroport international de Port-au-Prince en a fait la principale porte du pays pour l'aide internationale. Contrairement à l'essentiel du territoire urbain, cette commune n'est pas située dans une zone au relief marqué. Elle correspond au contraire au lit majeur de la Rivière Grise. Située sur un terrain plat (quoiqu'inondable), encore partiellement urbanisable au moment de l'occurrence du séisme, Tabarre a donc vu un grand nombre d'institutions internationales, d'ONG et de camps de déplacés s'installer sur son territoire (54 000 déplacés en octobre 2010 - COOPI, IRD, 2011). Aujourd'hui peuplée d'environ 124330 habitants $^{7}$, il s'agit d'un territoire stratégique pour le fonctionnement et le développement de la ville, d'où le choix opéré pour ce projet de recherche ${ }^{8}$.

Illustration 2 - Zones inondables de Tabarre (Port-au-Prince)

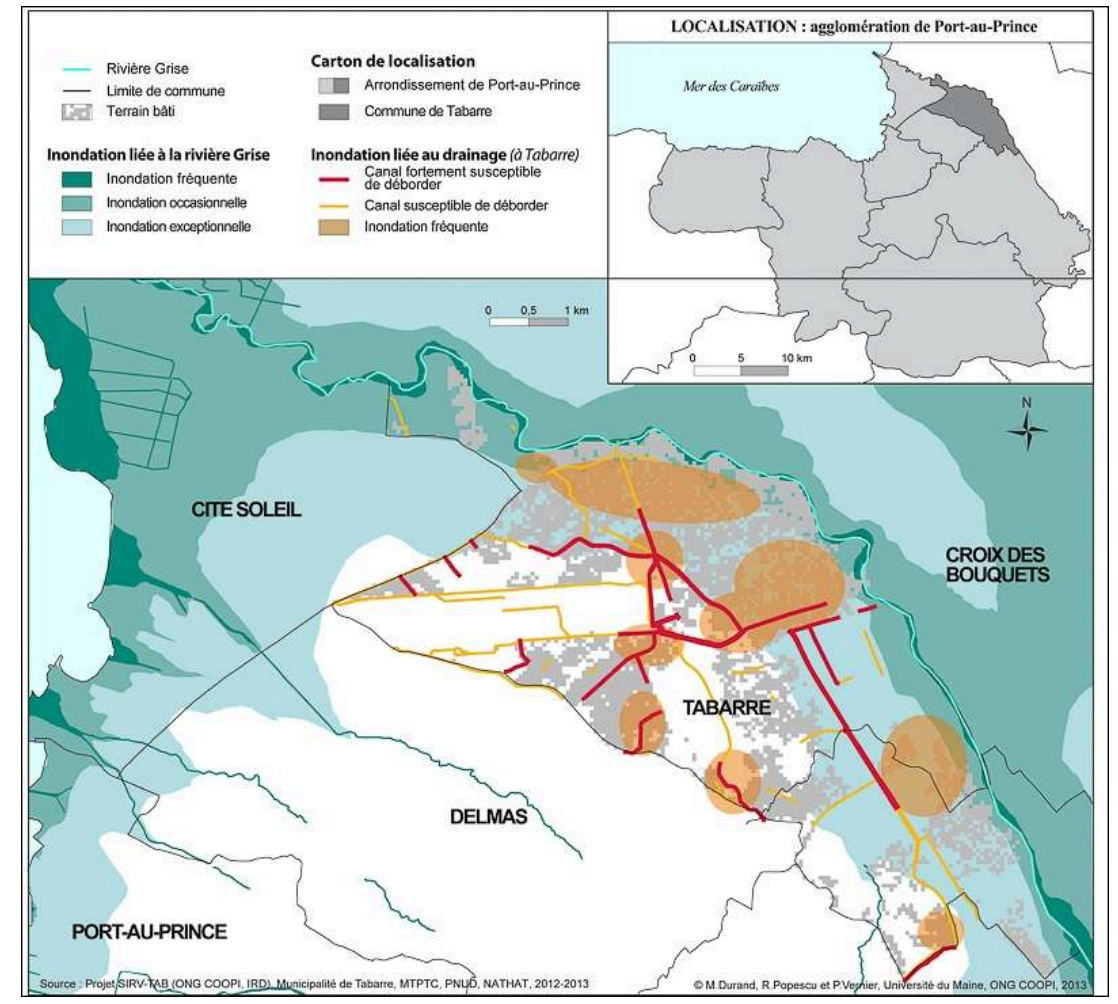

Sources: Projet SIRV-TAB (ONG COOPI, IRD), Municipalité de Tabarre, MTPTC, PNUD, NATHAT, 2012-2013 ; auteurs : M. Durand, R. Popescu et P. Venier, Université du Maine, ONG COOPI, 2013.

\section{Une attention très récente sur les retours d'expérience internationaux}

10 Avant de revenir sur la situation de crise qu'a connue Haïti, nous noterons que les retours d'expériences internationaux font l'objet d'une attention très récente, tout comme l'analyse de la gestion des déchets dans les catastrophes passées. Elle reste encore peu appropriée par les acteurs du secteur. Certaines grandes idées émergent toutefois de ces retours d'expériences. Les dernières crises ont fait l'objet de rapports ${ }^{9}$ cherchant à comprendre comment les déchets ont été gérés et soulignant des pistes 
d'actions possibles. Certaines idées permettent d'éclairer l'approche spatiale de la gestion des déchets post-catastrophe que nous cherchons à développer.

La première recommandation est de ne pas encombrer le système de gestion des déchets quotidiens. Les recommandations des acteurs ayant dernièrement eu à gérer des crises insistent pour concentrer, autant que possible, la gestion des déchets postcatastrophe dans des installations et des infrastructures spécifiquement dédiées à cela. Il s'agit de ne pas compromettre le fonctionnement quotidien du traitement des déchets ménagers, ce qui pourrait provoquer de graves dysfonctionnements dans la gestion ultérieure de la ville. L'objectif est également de ne pas compromettre les capacités de redémarrage économique d'un territoire en hypothéquant les capacités futures à fonctionner.

12 La seconde recommandation insiste sur la nécessité de mettre en place des sites de transit provisoires et intermédiaires pour gérer les déchets. Suite à une catastrophe, quoiqu'il arrive, ces sites de transit se mettent en place, de façon spontanée et non sécurisée ${ }^{10}$. Il semble donc plus judicieux d'essayer d'anticiper et de planifier leur implantation, afin qu'elle soit la plus adaptée possible. Dans l'urgence, aucune institution (quel que soit son niveau d'importance) ne prend le temps d'apporter les déchets sur une décharge située à plusieurs kilomètres de la ville. Il faut des points de collecte capables d'accueillir les déchets à distance raisonnable des lieux d'évacuation, sans pour autant engendrer de nouveaux risques du fait de mauvaises conditions sanitaires et environnementales. Ces points de stockage intermédiaires pourront alors faire l'objet d'un traitement postérieur, avec un appui éventuel de l'aide internationale.

Enfin, les rapports étudiés mettent en évidence que l'ensemble de ces mesures doivent être planifiées avant la catastrophe. Leur territorialisation doit également être abordée, sans pour autant figer strictement la localisation de chaque activité. Se positionner dans l'hypothèse d'une nouvelle catastrophe n'est guère aisé dans un pays, comme Haïti, récemment affecté par un grand tremblement de terre. La probabilité élevée de survenue de petites et moyennes catastrophes (liées par exemple aux ouragans et aux inondations) invite cependant à cette planification.

\section{Gérer les déchets à travers la relégation}

\section{La relégation et le sacrifice d'espace comme principe de gestion}

14 L'approche spatiale de la gestion des déchets mobilise le principe de relégation, c'est à dire d'éloignement $d u$ déchet par rapport à son lieu de production. Ce terme est ici compris dans l'acception classique des études de rudologie constatant le rejet des déchets dans des espaces déclassés (Gouhier, 2000). Cet éloignement physique des déchets revêt cependant plusieurs volets. Les autorités haïtiennes l'appliquent officiellement de façon stricte en cherchant à éloigner autant que possible les déchets, avec la création d'une décharge municipale au nord de l'agglomération (cf. illustration 3). Cette relégation, s'appuyant sur un espace trop éloigné de la zone urbaine, est toutefois peu mise en œuvre par les institutions et individus se chargeant de l'évacuation des déchets. Les déchets, qu'ils soient municipaux ou post-catastrophe, sont peu nombreux à terminer dans la décharge officielle de Truitier - Cité Soleil (Bras, 2010). Comme dans la plupart des villes en développement, une partie des déchets échappe au contrôle des autorités. Ils sont soit brûlés, soit évacués vers des espaces 
vides (terrains vagues, berges de cours d'eau, etc.), soit valorisés par tout un circuit informel d'acteurs. L'absence de traitement officiel ne signifie toutefois pas l'absence de valorisation ; le constat est d'ailleurs très souvent inverse (Durand, 2012).

Pour faire face à cette défaillance de la collecte des déchets solides, des logiques de relégation plus locales se mettent alors en place. Il ne s'agit pas d'une relégation à l'échelle de la ville, telle que celle mise en place par les autorités avec la décharge contrôlée, mais d'une relégation de proximité. Celle-ci n'est pas planifiée. Elle est le fruit de pratiques spontanées qui éloignent les déchets des quartiers les ayant produits, sans pour autant les faire sortir de la ville. Il s'agit de sacrifier des espaces (de taille très diverse), à l'intérieur même de la ville : des terrains non occupés, des bords de routes, des canaux, des ravines, etc. La localisation de ces dépôts répond alors à deux logiques. La première est la disponibilité des terrains et le fait qu'ils ne soient pas appropriés pour d'autres usages. Il peut s'agir de terrains très petits (la chaussée d'une route) ou plus vastes (les bords de la Rivière Grise). La seconde logique correspond à une certaine proximité et accessibilité du terrain. Les déchets sont déposés au plus proche du lieu de production, afin de ne pas effectuer un déplacement trop grand pour avoir à s'en débarrasser. Les entrées de quartiers, au croisement avec les avenues principales font alors souvent office de dépôts de déchets. Il s'agit alors simplement d'éloigner la nuisance de son espace de vie. La relégation est, dans ce cas, observée comme un double «éloignement social et spatial " (Neives de Oliveira, 2013), dans des lieux éloignés spatialement, quoique répondant à une certaine proximité. Ces espaces font l'objet d'une marginalisation spatiale (Sierra, Tadié, 2008) qu'il faut appréhender à différentes échelles suivant que la logique est locale ou métropolitaine.

En dehors des situations de crise, les villes ont toujours eu tendance à sacrifier certains espaces pour y déposer leurs déchets et faire fonctionner le système urbain. À l'échelle mondiale, ce n'est que depuis la deuxième moitié $d u \mathrm{XX}^{\mathrm{e}}$ siècle que des mécanismes de traitement et de valorisation des déchets ont été mis en place (Berdier et Deleuil, $2010)^{11}$. Le constat du sacrifice d'espace s'accroit alors en situation de crise. Il s'agit donc de comprendre quelles sont les logiques spatiales qui amènent à créer des espaces de relégation, afin de mieux anticiper sur une gestion des déchets post-catastrophe. Une telle connaissance permettrait de mieux préparer ce «sacrifice d'espace ». Puisque ce dernier semble inévitable en cas de grosse catastrophe, comme nous le détaillerons par rapport au séisme de 2010, il est plus judicieux d'essayer de l'anticiper afin d'en limiter au maximum les impacts négatifs. 
Illustration 3 - Espaces de gestion des déchets ménagers à Tabarre

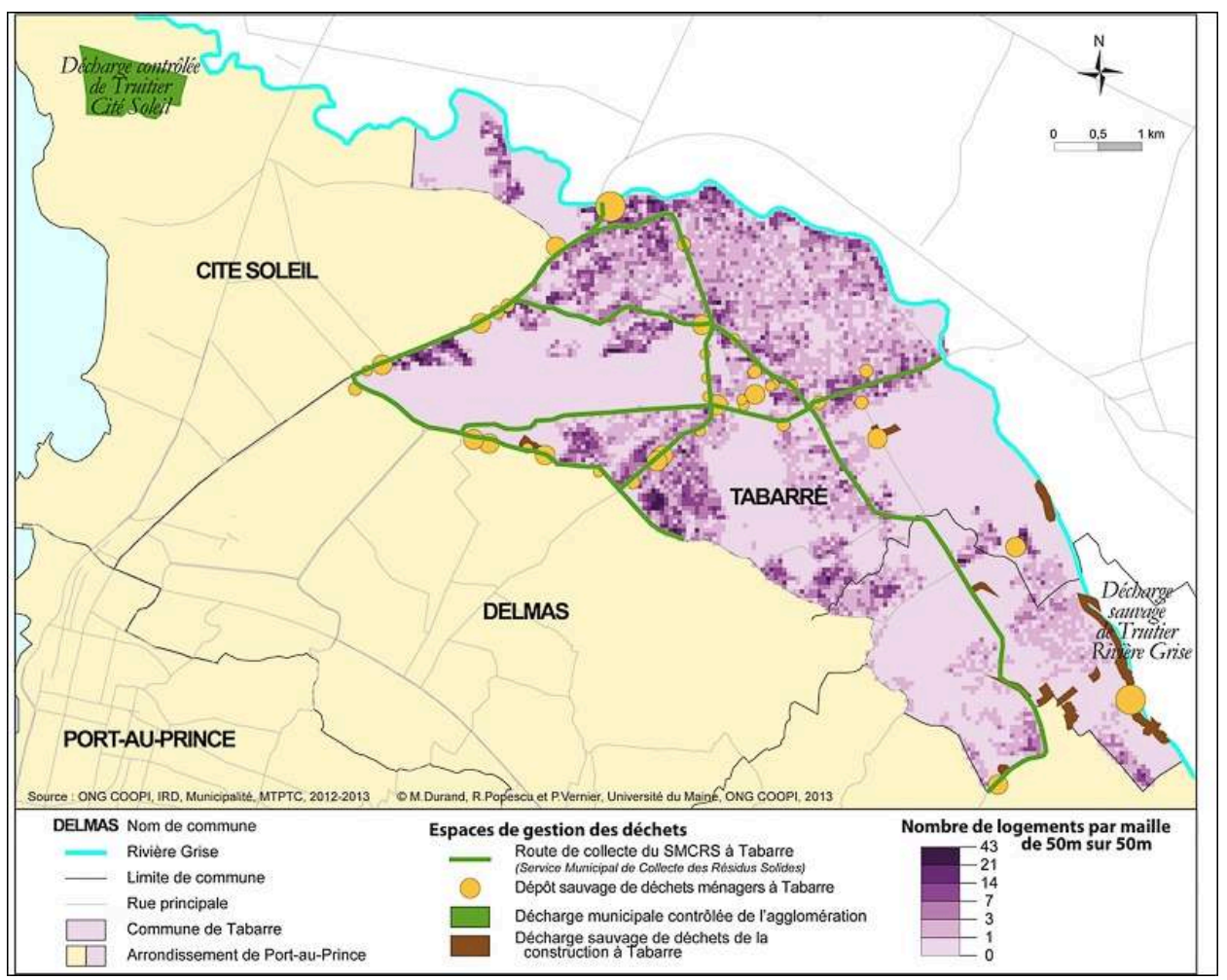

Sources : ONG COOPI, IRD, Municipalité, MTPTC, 2012-2013; auteurs : M. Durand, R. Popescu et P. Venier, Université du Maine, ONG COOPI, 2013

\section{La gestion des déchets ménagers et assimilés à Tabarre}

Pour comprendre la gestion des déchets post-catastrophe qui est faite à Port-au-Prince, en prenant en exemple la commune de Tabarre, il faut au préalable observer celle des déchets ménagers ainsi que des déchets de la construction.

De manière générale, les déchets de la zone métropolitaine de Port-au-Prince, sont gérés par le SMCRS ${ }^{12}$ et les mairies des communes faisant partie de l'agglomération (Samper et al., 2006 ; Lombart et al., 2014). L'espace officiellement désigné pour recevoir tout type de déchets est la décharge de Truitier, dans la commune de Cité Soleil. Du fait d'une capacité insuffisante des acteurs publics à collecter tous les déchets, ceux-ci terminent sur différents types d'espaces selon des logiques différentes. En termes d'accessibilité, on peut noter que les espaces de dépôts des déchets ménagers répondent à une logique spatiale spécifique. Ils sont tous situés le long des principales voies de communication ${ }^{13}$, là où la population passe quotidiennement et jette ses déchets. Ensuite, le SMCRS collecte les déchets sur ces avenues, sans jamais rentrer à l'intérieur des quartiers. Il est important de noter que ces logiques spatiales existaient déjà avant le séisme de 2010. Les quantités limitées de déchets ménagers (en comparaison aux déchets de la construction par exemple - cf. ci-dessous), permettent un dépôt au bord de voies de communication au cœur de la ville. 
Illustration 4 - Dépôt sauvage des déchets ménagers en bord de route à Tabarre

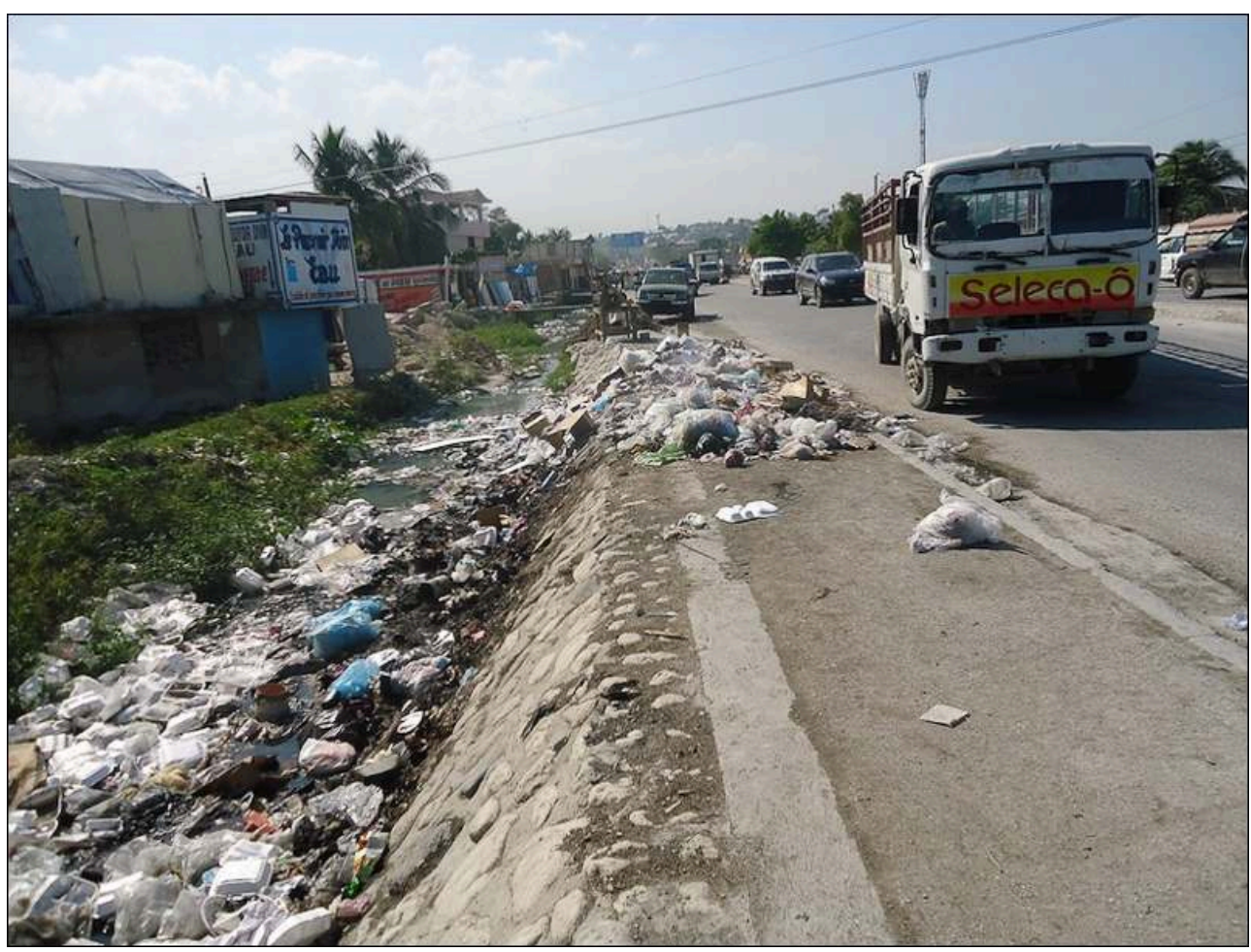

Auteur : R. Popescu, 2013

\section{Les déchets de la construction : des matériaux inertes en grande quantité}

19 Les déchets de la construction ne se caractérisent pas non plus par une gestion publique efficace. S'ils sont de la responsabilité des entreprises les ayant produits, ils ne font l'objet d'aucun traitement (la situation est d'ailleurs similaire dans la plupart des pays du monde, au sud comme au nord). Ils sont donc également rejetés sur des décharges sauvages, aux caractéristiques distinctes de celles des déchets ménagers. Ils occupent des espaces plus grands, du fait de leurs volumes importants. Ils ne peuvent donc pas se concentrer uniquement le long des voies de communication et à l'entrée des quartiers d'habitation. Ils sont essentiellement évacués vers des espaces disponibles plus vastes, plus éloignés, des terrains vagues, des bords de rivières, des ravines inhabitées, etc. Leur poids fait que leur évacuation nécessite un véhicule, permettant ainsi de les transporter plus loin que les déchets ménagers. La concentration des déchets de la construction est donc plus grande dans des lieux plus ciblés. La logique de proximité veut que le rejet de ce type de déchets se fasse au plus proche du lieu de production. La logique de relégation, au contraire, les éloigne et restreint le nombre de lieux concernés. Le coût du transport et la disponibilité de terrains sont alors les critères importants. La gestion informelle des déchets de la construction, réalisée par des micro-entreprises, est très présente. Il serait donc nécessaire d'approfondir l'étude anthropologique des choix d'espaces au niveau très localisé pour comprendre le processus de constitution d'une décharge, au-delà de la seule approche spatiale et technique. 
logiques spatiales sont similaires à celles observées dans toutes les villes du monde en dehors des situations de crise (Gouhier, 2003). Mais en temps de crise, l'exemple d'Haïti montre que ces logiques sont exacerbées. L'apparition de ces différents types d'espaces est le résultat d'une pratique de relégation de proximité. Les capacités techniques de collecte et de transport des déchets jusqu'à la décharge officielle étant limités, ces espaces sont considérés de proximité dans la mesure où ils permettent un rassemblement des déchets à une distance minimale. La manière dont ils se mettent en place sur le territoire dépend de plusieurs facteurs dont les plus importants sont la disponibilité de l'espace, l'accessibilité, le type de déchets, la distance entre le lieu de production et la décharge, le coût lié au transport et la perception de la population sur la question de la propreté.

Illustration 5 - Décharge sauvage de déchets de la construction en bord de la Rivière Grise, Tabarre

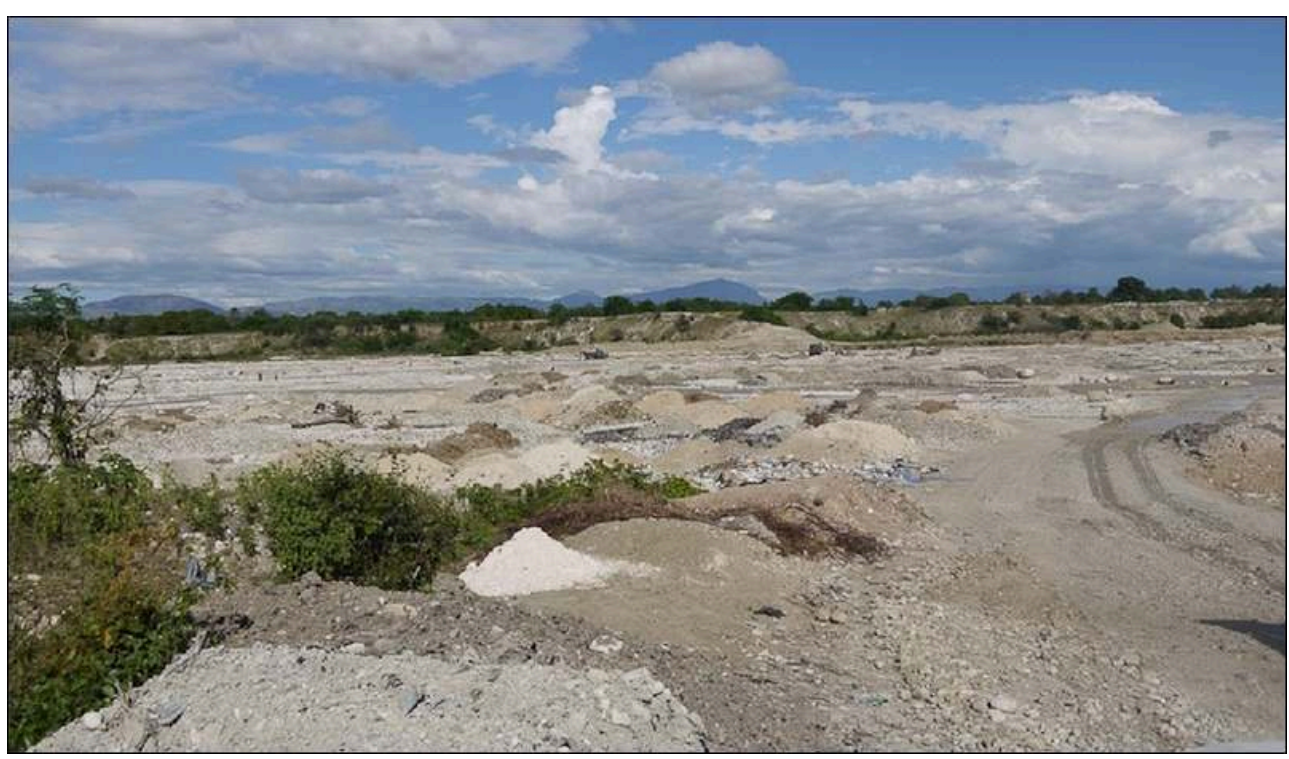

Auteur : R. Popescu, 2012

\section{Structuration spatiale de la gestion des déchets post- catastrophe}

21 Après le tremblement de terre d'Haïti en 2010, les décharges sauvages existantes ont reçu une partie importante des déchets de la catastrophe. C'est particulièrement le cas des décharges qui recevaient déjà des déchets de la construction avant le séisme (répertoriées comme " décharges sauvages de déchets de la construction à Tabarre " sur l'illustration 3). Une grande quantité de décombres est progressivement venue s'ajouter aux déchets de ces sites. Les débris provenant des maisons détruites étant très proches des caractéristiques des déchets de la construction (pour l'essentiel des déchets inertes), leur gestion a été similaire. Les logiques spatiales de gestion des déchets ménagers et de la construction observées précédemment, sont alors exacerbées en situation de crise. Il est également possible d'observer la création de nouvelles décharges sauvages directement liées au séisme, dont l'implantation est également analysable spatialement. 


\section{La difficile traçabilité des déchets produits par le séisme}

Suite à la catastrophe de 2010, la question des déchets a été parmi les dernières à être prise en compte, plusieurs mois après le séisme. Selon les estimations des NationsUnies, le séisme, à l'origine de plus de 200000 morts et de 1,5 millions de sans-abris, a produit environ 10 millions de $\mathrm{m}^{3}$ de débris (UNOPS, 2011). Il est difficile d'obtenir des informations fiables sur ces volumes et sur la façon dont ils ont été enlevés. Plusieurs acteurs sont intervenus dans cette phase d'enlèvement, encadrée par deux plans successifs des forces onusiennes (celles de la MINUSTAH - Mission des Nations Unies pour la Stabilisation en Haïti) :

- Les acteurs de l'aide internationale ont enlevé environ 31,5\% des décombres (PNUD, 2012). C'est grâce à ces acteurs qu'il existe aujourd'hui une traçabilité des débris collectés, même si elle ne couvre pas tout le volume enlevé. L'aide internationale regroupe des forces militaires et civiles des Nations Unies déjà sur place avant le séisme, des États étrangers, ainsi que de nombreuses ONG internationales. L'aide massive et réactive apportée par ces acteurs, leurs moyens techniques et humains importants, ont laissé néanmoins de nombreux acteurs haïtiens sceptiques sur l'efficacité d'une action s'appuyant peu sur les forces locales.

- Les acteurs publics haïtiens ont enlevé environ $11 \%$ des déchets post-catastrophe. Ce sont principalement les services de l'État dépendant du Ministère des Travaux Publics des Transport et des Communications (MTPTC). Les municipalités ont également apporté leur contribution. Cependant, malgré des financements ponctuels reçus de la part de l'aide internationale pour améliorer leur fonctionnement, leur rôle dans l'évacuation des débris n'a pas été très important en raison de moyens techniques limités et souvent en mauvais état.

- La société civile et le secteur informel ont enlevé le reste des déchets, soit $57,5 \%$ du volume total produit par le tremblement de terre. Selon les chiffres publiés par le PNUD, cette catégorie inclut les déchets enlevés par les propriétaires des bâtiments $(27,5 \%)$ et les déchets dont le devenir est «inconnu ${ }^{14}(30 \%)$. Dans tous les cas, ils ont été pris en charge par les occupants des maisons effondrées, soit directement, soit à travers de petits entrepreneurs du secteur du bâtiment et des travaux publics. Dans certains cas ces acteurs ont récupéré les déchets comme matériaux pour entamer la reconstruction (notamment réaliser des remblais et des fondations). Cette gestion reste essentiellement informelle et aléatoire puisqu'une partie des décombres, encore visibles sur l'espace public, n'a en réalité pas encore été enlevée. Une part également importante a été réutilisée directement sur place comme matériaux de construction. Le MTPTC milite et communique pour un usage raisonné de ces matériaux, afin qu'ils ne soient réutilisés que dans des constructions non structurantes, pour éviter le manque de solidité des bâtiments. Son discours ne semble pourtant pas faire l'objet d'un écho important à Port-au-Prince.

Parmi l'ensemble des déchets collectés, il est important de noter que seuls $6 \%$ ont terminé dans l'un des trois centres officiellement habilités pour les recevoir (identifiés sur l'illustration 6) comme " centre d'enfouissement et de valorisation officiel»). Ces centres ont tous été mis en place suite au séisme sous la coordination de la MINUSTAH, en sous-traitant leur gestion à des ONG ou des entreprises. Les deux premiers à avoir été créés sont situés à proximité du centre de Port-au-Prince, afin d'être au plus près des gisements de déchets. Ils sont cependant de taille très réduite. Le troisième a été créé à l'intérieur de la décharge contrôlée déjà existante de Truitier-Cité Soleil. Si ces centres sont théoriquement temporaires (puisque sans utilité une fois tous les déchets post-catastrophe traités), ils semblent aujourd'hui se pérenniser. Tous les trois gérés 
par des entreprises ou des ONG étrangères, ils ont l'obligation de leurs bailleurs de fond de recycler les déchets collectés. Si les infrastructures ont été mises en place pour cela, l'absence de structuration des filières n'a pas permis de réel recyclage organisé ${ }^{15}$. Le seul recyclage effectué est celui opéré par les propriétaires de constructions ou les petites entreprises du BTP ayant récupéré les décombres comme matières secondaires.

\section{Logiques spatiales de dépôt des déchets post-catastrophe}

La carte de l'illustration 6 représente les différents espaces formels et informels où les décombres issus du tremblement de terre ont été déposés. Elle met en évidence les espaces les plus importants au niveau métropolitain, en réalisant un travail de détail plus fouillé sur la commune de Tabarre. Les déchets post-catastrophe étant essentiellement des déchets inertes, similaires aux déchets de la construction, ce sont aujourd'hui, plusieurs années après le séisme, ces déchets qui sont toujours visibles. La représentation du volume de déchets collectés par section communale met en avant le rapport entre les espaces densément peuplés et le volume de débris produits et donne une idée de la distance qu'il y a entre les espaces les plus importants en termes de production de débris et les différents types de décharges qui les ont accueillis.

Illustration 6 - Densité de l'habitat et des débris produits par le séisme de 2010 à Port-au-Prince

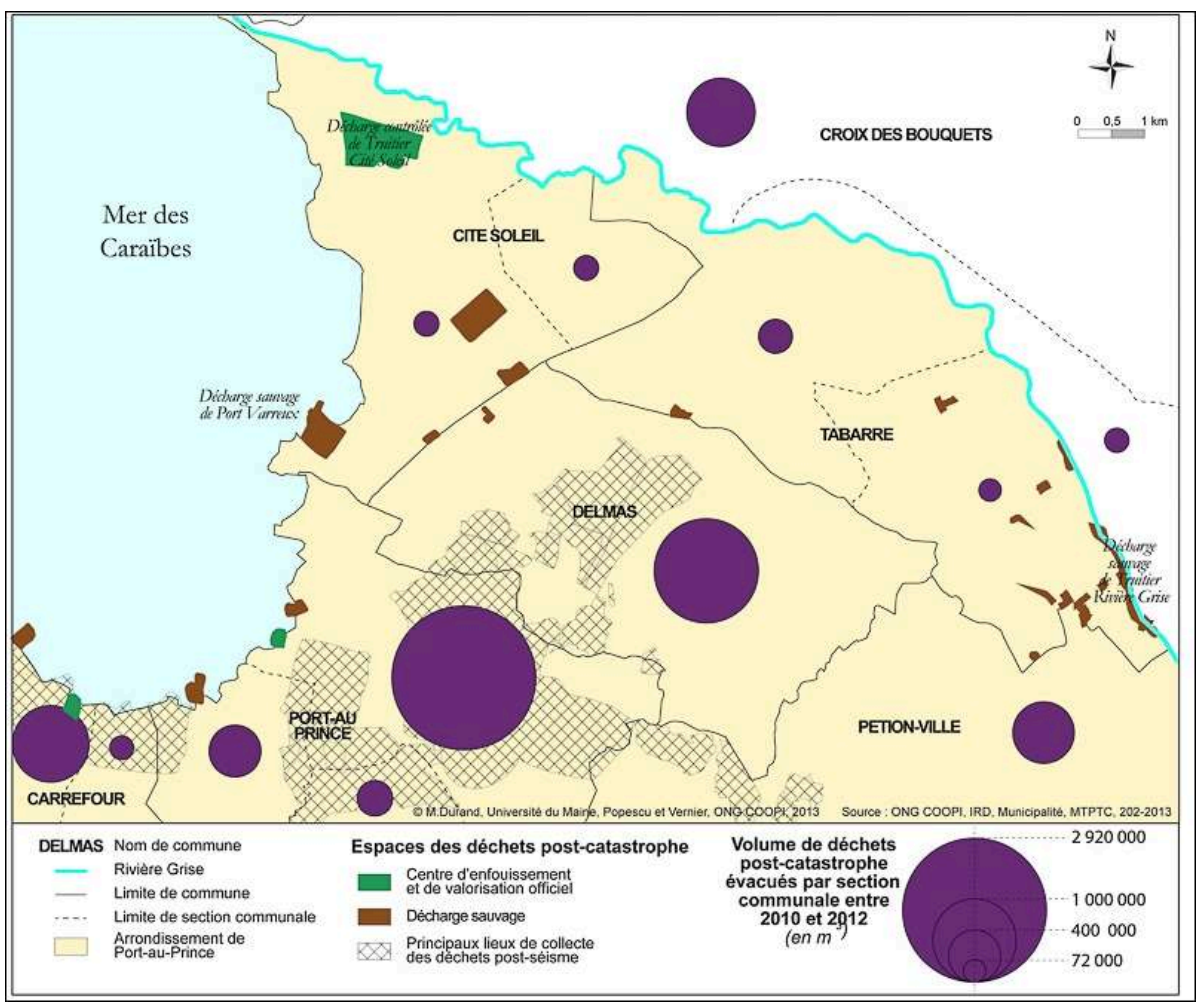

Sources : ONG COOPI, IRD, Municipalité, MTPTC, 2012-2013 ; auteurs : M. Durand, R. Popescu et P. Venier, Université du Maine, ONG COOPI, 2013.

On observe ainsi deux zones principales de concentration pour les déchets du séisme : le littoral et les bordures de la Rivière Grise. Cette logique de distribution observée à l'échelle métropolitaine suit les mêmes logiques spatiales qui ont abouti à la création des décharges de déchets ménagers et de la construction. Ces espaces sont assez 
naturellement apparus comme exutoires pour les déchets. Tout en étant libres, ils sont plus proches des communes denses de la ville où ont été produits les débris que ne l'est la décharge officielle de Truitier Cité-Soleil. Celle-ci, quoiqu'habilitée à recevoir les déchets du séisme ${ }^{16}$, est située trop loin du cœur de la ville pour que les collecteurs de déchets s'y rendent. Les décharges sauvages sont ainsi souvent situées sur le chemin emprunté par les camions pour aller des zones de production de déchets vers la décharge de Truitier. On peut ainsi émettre l'hypothèse que les déchets des décharges $\mathrm{du}$ littoral proviennent davantage du centre-ville de Port-au-Prince (car elles sont situées sur la route entre le centre-ville et la décharge officielle), alors que ceux des décharges de la Rivière Grise peuvent venir des quartiers aisés de Pétion-ville et de Delmas (également sur la route vers la décharge officielle) ${ }^{17}$. Les limites communales semblent également plus à même de recevoir ces déchets, probablement du fait du manque d'attention des pouvoirs publics locaux sur ces marges administratives.

Il existe aussi d'autres facteurs importants qui expliquent cette répartition comme la disponibilité foncière, la relative proximité et l'accessibilité des terrains par rapport aux zones les plus affectées par le séisme, le coût lié au transport et au stockage des décombres, la proximité d'espaces aquatiques (rivière ou côte) ou encore les pratiques de gestion des déchets avant le séisme. Les déchets permettent ainsi de remblayer des zones inondables puisqu'ils ont été principalement déposés le long de la rivière et de la côte. Comme le montre l'illustration 6 , ces espaces sont situés dans la périphérie nord et ouest de l'agglomération ${ }^{18}$, c'est-à-dire en dehors des zones principalement détruites par le tremblement de terre (le centre de Port-au-Prince), mais sur des terrains disponibles. En tenant compte de la localisation géographique de Tabarre dans une plaine inondable et de la fréquence des inondations, la même logique peut s'appliquer pour le sud-est de Tabarre.

\section{La création spontanée d'espaces intermédiaires de dépôt de proximité}

L'analyse spatiale de la gestion des déchets post-catastrophe de Tabarre permet donc de mettre en avant les logiques de relégation. On observe ainsi qu'en l'absence de capacités de traitement des déchets, les pratiques opérées pour leur gestion visent à déplacer la nuisance. Les acteurs impliqués dans l'enlèvement des déchets vont également faire le choix de leur exutoire en fonction des moyens de transport dont ils disposent. Une grande entreprise pourra aller plus loin grâce à un camion, quand un petit acteur informel aura la capacité à s'infiltrer à l'intérieur de la ville, difficilement accessible aux grands véhicules du fait de l'encombrement des rues par les décombres. L'étape technique du transport des déchets a abouti à ce déplacement. Or celui-ci ne se fait pas de façon aléatoire. Il est le résultat des capacités différenciées des acteurs qui interviennent dans la gestion (population et secteur informel), ainsi que d'une logique spatiale alliant les concepts parfois contradictoires de relégation, de proximité et de disponibilité foncière. Les déchets ont ainsi, pour l'essentiel, été déposés dans des espaces non prévus à cet effet (à l'exception des $6 \%$ terminant dans les trois centres officiels). Il s'agit d'espaces de dépôt intermédiaires improvisés, afin de palier à l'éloignement de la décharge officielle de Tabarre. Ils se sont constitués en répondant aux concepts cités ci-dessus, par absence d'autre perspective pour un dépôt plus structuré des déchets post-catastrophes. 
Les constats opérés à Tabarre vont donc dans le même sens que les retours d'expériences internationaux, préconisant la constitution d'espaces de dépôt intermédiaires, afin d'organiser le dépôt de proximité. Il peut alors sembler judicieux de chercher à anticiper et à encadrer la délimitation de ces espaces qui se mettront en place quoiqu'il arrive, avec ou sans planification en cas de catastrophe. Les conditions d'adéquation de ces lieux ne pouvant raisonnablement pas être celles d'une décharge contrôlée, il faut alors insister sur leur aspect provisoire et sur l'obligation initiale de réfléchir à leur démantèlement postérieur. Le besoin de proximité dans la gestion des déchets post-catastrophe fait l'objet d'un constat partagé par tous sur plusieurs terrains internationaux, son anticipation, même dans des conditions environnementales dégradées, va donc dans le sens d'une baisse de la vulnérabilité des populations affectées et du système de gestion de crise en place. Ce point de vue rejoint les résultats des recherches géographiques récemment menées qui insistent sur l'importance de l'adéquation spatiale et fonctionnelle entre les ressources de la gestion de crise et les besoins exprimés notamment par la population (D'Ercole et Metzger, 2009 ; D'Ercole et al., 2012). De même, l'anticipation de l'occupation du sol en vue d'une période d'urgence, comme facteur de réduction de vulnérabilité, a été mis en évidence dans de nombreux cas : celui des zones de dépôt de déchets post-catastrophe, mais également pour les zones de camps de déplacés, d'extension d'hôpitaux ou encore de stockage de l'aide alimentaire internationale (D’Ercole et al., 2011).

\section{Conclusion}

Une fois établie la nécessité d'anticiper la gestion des déchets post-catastrophe, nous pouvons analyser plus en détail leur gestion suite au séisme d'Haïti en 2010. L'analyse de cette gestion sous un angle spatial permet d'identifier des logiques assez récurrentes dans le domaine des déchets. En effet, pour éliminer au mieux les déchets produits par la catastrophe, la principale méthode utilisée fut celle de la relégation, c'est-à-dire de l'éloignement des déchets en dehors de la ville. Dans les faits, cet éloignement s'opère à une échelle beaucoup plus fine, sur un territoire infra-métropolitain beaucoup plus restreint. On retrouve donc la double logique spatiale de relégation et de proximité, puisque les déchets sont déposés sur les premiers terrains disponibles répondant à des critères d'accessibilité, de disponibilité foncière et de non concurrence avec les pratiques locales. Il s'agit là de logiques observées dans la gestion des déchets quotidiens de beaucoup de villes des pays du sud (Florin, Cirrelli, 2014).

30

Certaines observations sont cependant davantage liées à la spécificité des déchets postcatastrophe: des volumes importants et devant être gérés dans un laps de temps relativement court (à Port-au-Prince, les déchets ont été gérés dans les trois ans ayant suivi le séisme). Il est ainsi illusoire d'imaginer un espace de dépôt, voire de recyclage de ces déchets, trop éloigné de la ville. Il ne sera en effet utilisé que partiellement. On observe d'ailleurs la même situation dans les grandes métropoles du monde, trop grandes pour qu'un seul exutoire puisse constituer l'unique point de sortie. Il faut donc imaginer des centres de transfert, où les déchets pourraient être entreposés provisoirement, avant d'être évacués, plus tard, vers le centre officiellement habilité à les traiter (élimination ou valorisation). Ce type de centre de transfert s'est mis en place spontanément à Port-au-Prince, sur les espaces identifiés dans cet article. Les risques liés à l'absence de gestion de ces espaces sont alors nombreux (inondations, 
pollution des ressources en eau, risques sanitaires, etc.). Il semblerait donc pertinent de réfléchir à une planification de ces espaces tout en admettant que la gestion des déchets ne peut être optimale dans la phase de gestion de crise. La constitution d'espaces intermédiaires de dépôt provisoire permettrait ainsi de structurer le territoire, mais également de structurer les acteurs intervenant dans le domaine.

31 Par ailleurs, les résultats de la recherche effectuée à Tabarre ont permis de montrer qu'il existe des liens très étroits entre les différentes ressources de la gestion de crise : secours, refuges, eau, alimentation, soins d'urgence, etc. (COOPI, IRD, 2013). Toutes ces activités nécessitent en effet du foncier disponible. Une concurrence pour l'espace se met alors en place, particulièrement problématique dans une ville aussi dense que Portau-Prince. Toute la question réside alors dans les moyens à déployer pour arriver à cette maîtrise de l'espace, dans un pays où toute planification urbaine est impossible. Il est difficilement imaginable de réserver des terrains, de les mettre hors la ville, dans l'attente d'une hypothétique catastrophe. Il semble plus pertinent de réfléchir à des critères permettant d'identifier ces espaces de façon à avoir une lecture dynamique et rapide du territoire en cas de catastrophe majeure. Les moyens légaux d'appropriation de ces espaces (même dans un contexte où propriété foncière et utilisation d'un terrain ne vont pas toujours de pair) peuvent également permettre d'anticiper sur les situations d'urgence.

Les espaces de dépôt des déchets post-catastrophe et leur gestion font donc pleinement partie des ressources de la gestion de crise. Ce thème est généralement inscrit dans les phases de récupération et de reconstruction, puisqu'il s'insère dans le redémarrage $d u$ fonctionnement dit normal de la ville. Il mériterait cependant d'être pensé plus en amont en termes de préparation et de planification préventive en considérant notamment la concurrence foncière entre les besoins des différentes ressources de la gestion de crise.

\section{BIBLIOGRAPHY}

Barles S., 2005. L'invention des déchets urbains, France: 1790, 1970. Paris, Champ vallon, 297 p.

Béraud H., Jadot J., Barroca B., Hubert G., Bauduceau N., 2012. Estimation du volume et de la nature des déchets produits par une inondation : éléments de réflexion pour l'élaboration d'une méthodologie. $12^{\text {th }}$ Congress Interpraevent, Grenoble, $12 \mathrm{p}$.

Berdier C., Deleuil J.-M., 2010. Le système « ville-déchet », une mise en perspective historique. In Dorier-Apprill E., Ville et environnement, Paris, Sedes, p. 453-466.

Bozza A., 2012. Modélisation du risque de ruissellement et inondation à Port au Prince. Toulouse, Université de Toulouse, Rapport de stage, $56 \mathrm{p}$.

Bras A., 2010. Éléments pour une définition de la problématique de la propreté urbaine en Haïti : le cas de Port-au-Prince. Thèse de doctorat, Lyon, INSA, $213 \mathrm{p}$. 
Brown C., Milke M., Seville E., 2011. Disaster waste management: a review article. International journal of integrated waste management, science and technology, $57 \mathrm{p}$.

COOPI, IRD, 2011. Étude de vulnérabilité de la commune de Tabarre. Analyses des enjeux majeurs et des facteurs de vulnérabilité. Port au Prince, Haïti, 321 p.

COOPI, IRD, 2013. Ressources de gestion de crise concernant Tabarre, volume 3 du rapport final du projet $S I R V-T A B$ «Création d'un Système d'Information sur les ressources et les vulnérabilités pour la préparation aux catastrophes, la gestion des crises et la gestion préventive du territoire de Port-au-Prince, Haïti ». Portau-Prince, Haïti, 623 p.

D’Ercole R., Metzger P., 2009. Las dimensiones espaciales del manejo de crisis. Interés de la investigación y aplicación en Quito. In D'Ercole R. (ed.), Hardy S. (ed.), Metzger P. (ed.), Robert J. (ed.), Vulnerabilidades urbanas en los paises andinos (Bolivia, Ecuador, Peru), Bulletin de l'Institut Français d'Etudes Andines, 38 (3), p. 893-916.

D’Ercole R., Metzger P., Robert J., Hardy S., Gluski-Chraibi P., Vernier P., Sierra A., Perfettini H., Guillier B., 2011. Recursos de respuesta inmediata y de recuperación temprana ante la ocurrencia de un sismo y/o tsunami en Lima Metropolitana y Callao. Estudio SIRAD, Proyecto "Preparación ante desastre sísmico y/o tsunami y recuperación temprana en Lima y Callao”, ECHO/PNUD/INDECI/ COOPI/IRD, Lima, $189 \mathrm{p}$.

D’Ercole R., Hardy S., Metzger P., Robert J., Gluski-Chraibi P., 2012. Les dimensions spatiales et territoriales de la gestion de crise à Lima. Vertigo, vol. 12, $\mathrm{n}^{\circ}$ 1, [En ligne], http:// vertigo.revues.org/12009

De Oliveria Neves F., 2013. Gestão pública de resíduos sólidos urbanos: problemática e práticas de gestão no oeste paranaense. Thèse de doctorat, Curitiba, Universidade Federal do Paraná, 279 p.

Durand M., 2012. La gestion des déchets dans les pays en développement : comment tirer profit des difficultés actuelles? Revue Flux, $\mathrm{n}^{\circ} 87$, « Mutation des services urbains : processus et enjeux ", p. 18-28.

Durand M., 2014. El manejo de residuos en Lima: vulnerabilidad, desigualdad y sostenibilidad. Lima: IFEA, IRD, $256 \mathrm{p}$.

Florin B., Cirrelli C. (dir.), à paraître. Sociétés urbaines et déchets : éclairages internationaux. Presses Universitaires François Rabelais.

Gouhier J., 2000. Au-delà du déchet, le territoire de qualité, manuel de rudologie. Rouen, Presses Universitaires du Rouen et du Havre, 240 p.

Gouhier J., 2003. De la décharge au territoire de qualité : évolution de la place des déchets dans la société. In Bertrand J.-R., Laurent F., De la décharge à la déchetterie, Rennes, PUR, p. 17-37

Lombart M., Pierrat K., Redon M., 2014. Port-au-Prince : un « projectorat » haïtien ou l'urbanisme de projets humanitaires en question. Cahiers des Amériques latines, 75, p. 97-123.

Ngo C., Regent A., 2008. Déchets, effluents et pollution, impacts sur l'environnement et la santé. Paris, Dunod, $178 \mathrm{p}$.

November V., Penelas M., Viot P., 2008. L'effet Lully : un territoire à l'épreuve d'une inondation. Revue Cosmopolite, $\mathrm{n}^{\circ}$ 17, p. 89-106.

PNUD, 2012. UN common debris projects: foundations for development. Port-au-Prince, PNUD, 3 p.

PNUD, 2013. Guide technique pour la gestion des débris, l'expérience haïtienne 2010-2012. PNUD, 37 p. 
ROBIN DES BOIS, 2007. Déchets post-catastrophe : risques sanitaires et environnementaux. Paris, GEIDE, ADEME, $300 \mathrm{p}$.

Samper O., Chapal E., Brailowsky A., 2006. Analyse de la problématique des déchets solides dans la zone métropolitaine de Port-au-Prince. Port-au-Prince, MTPTC, BID, 51 p.

Sierra A., Tadie J. (dir.), 2008. La ville face à ses marges. Bondy, IRD, Autrepart, nº45, 228 p.

UNOPS, 2011. Reconstruire Haïti, rapport annuel de 2011. UNOPS, 34 p.

\section{NOTES}

1. Étude réalisée dans le cadre du projet SIRV-TAB «Création d'un Système d'Information sur les Ressources et les Vulnérabilités pour la préparation aux catastrophes, la gestion des crises et la gestion préventive du territoire de Tabarre, Haïti » (août 2012 - décembre 2013), financé par le Département d'Aide Humanitaire et Protection Civile de la Commission Européenne (ECHO) et réalisé par l'ONG Cooperazione Internazionale (COOPI) et l'Institut de Recherche pour le Développement (IRD), en collaboration avec la Municipalité de Tabarre, la Direction Nationale de la Protection Civile (DPC) et le Centre National d'Information Géo-Spatiale (CNIGS).

2. L'ONG COOPI, qui a accueilli les auteurs de ce texte, est présente depuis 2011 à Tabarre, pour travailler sur les différentes phases successives à la gestion de crise issue du séisme de 2010. C'est dans ce cadre que l'IRD a pu y développer ses projets de recherche. Roxana Popescu y a ainsi fait plusieurs missions de plusieurs mois afin de collecter les données présentées dans ce texte. Robert D'Ercole y a également passé de nombreux mois afin de diriger le programme SIRV-TAB. Enfin, Mathieu Durand a appuyé l'équipe à distance sur la question des déchets, tout en y faisant une mission de quelques semaines.

3. Une autre publication des mêmes auteurs portera plus précisément sur l'analyse des rapports entre les acteurs, cherchant à fouiller les causes des modes de gestion décrits dans ce texte, ainsi que les éléments qui pourraient inviter ces acteurs à faire des choix différents dans le futur. Il aurait été trop long d'aborder l'ensemble de ces éléments dans ce même texte.

4. Les dénivelés intra-urbain vont de $1500 \mathrm{~m}$ à Kenscoff, à l'est de la ville, jusqu'au niveau de la mer sur la côte, à l'ouest.

5. Comme nous le verrons plus loin, en l'absence de collecte efficace, ces espaces sont des lieux privilégiés de réception des déchets solides de la ville.

6. Entretiens réalisés en janvier et février 2013. La méthodologie développée dans ce travail de recherche a été créée afin de répondre aux exigences du projet SIRV-TAB, c'est-à-dire identifier les ressources de la gestion de crise sur un terrain donné. Elle s'appuie sur les expériences du programme PACIVUR (Programa Andino de Capacitación e Investigación sobre riesgos y Vulnerabilidad en medio Urbano) mené depuis 1999 par l'IRD en Amérique Latine. Concernant le volet « déchets post-catastrophe » de cette étude, 33 entretiens ont été réalisés avec les acteurs publics et privés du territoire. Un SIG a également été monté afin de spatialiser ces ressources, sur la base d'analyse d'images satellites, d'observations de terrain et de données collectées lors des entretiens. Ce SIG s'est monté sur place grâce à l'appui de Pierre Vernier (IRD). Un atelier a enfin été organisé avec les responsables locaux (7 février 2013) afin de vérifier et d'affiner les données. 7. Estimations 2012 de l'IHSI (Institut Haïtien de Statistique et Informatique).

8. Au-delà de la volonté réelle de coopération des institutions locales, élément clef dans notre approche d'un projet de recherche-action.

9. Ces rapports sont recensés dans les analyses bibliographiques de Robin des bois, 2007, November et al., 2008 ; Brown et al., 2011 ; Béraud et al., 2012.

10. Nous l'illustrerons par la suite avec le cas haïtien. 
11. Sachant qu'avant la révolution industrielle, très peu de matière était considérée comme « déchet », puisque l'essentiel était valorisé avant d'atteindre ce stade (Barles, 2005).

12. Service Métropolitain de Collecte des Résidus Solides.

13. Le recensement de ces points n'a été effectué que sur le territoire de la commune de Tabarre (cf. illustration 3), dans le cadre du travail du projet SIRV-TAB, mais la logique est la même dans toute la ville.

14. Ce chiffre inclut une partie des déchets toujours visibles sur le terrain qui ont toutefois été pris en charge par la population pour être mis sur le côté des routes et dans les décharges sauvages.

15. Ce point fera prochainement l'objet d'une autre publication.

16. Cette décharge reçoit également les déchets ménagers de la ville. La partie «décharge municipale " ne fait l'objet d'aucun contrôle. Seul le secteur qui récupère les déchets de la construction est bien géré par plusieurs entreprises nord-américaines depuis le séisme.

17. Ces deux hypothèses ne peuvent pas être démontrées en l'absence de traçabilité des déchets déposés dans les décharges. Ils correspondent cependant à des logiques observées dans d'autres pays du sud (Durand, 2014).

18. On observe un phénomène similaire sur la côte sud-ouest de la ville, en dehors du champ de cette carte.

\section{ABSTRACTS}

Waste-disaster production is often considerate less urgent or less important than access to health or water. However, it may be central in recovery and reconstruction process of the affected area. It reduces negatives effects (pollution, canal obstruction, interrupted communications...) while participating in economic revitalization of this area (waste recycling, stimulating economic sectors...).

In this context, the article presents results of research carried out in the metropolitan area of Port-au-Prince and one of its districts, Tabarre, following the January 12, 2010 earthquake. The aim is to analyze spatial dimension of waste post-disaster management. This analysis permit to understand the destination of $94 \%$ of the 10 million $\mathrm{m} 3$ of earthquake-waste, that have not been sent to the official landfills and recycling center. It allows understanding the spatial logics waste management implemented by national and international authorities, such as civil society and informal sector. It correspond to relegation waste logical threw sacrificed spaces. This removal takes place at several scales, taking into account use landing and proximity between the waste fields and waste disposal sites. Existing logic of spatial household waste and construction waste management are exacerbating after the disaster.

The findings made in Port-au-Prince agree with international feedback about disasters. These advocate, as possible, anticipated management of post-disaster waste, especially where disasters are frequent, as in Haiti, including planning of landfills and temporary intermediate places for transit and sorting waste.

La prise en compte des déchets produits par une catastrophe, souvent considérée comme moins urgente, voire moins importante que l'accès à la santé ou à l'eau, peut se révéler centrale dans la récupération et la reconstruction du territoire affecté. Elle en réduit les effets négatifs (pollutions, obstruction de canaux, communications interrompues...) tout en participant à la 
redynamisation économique de ce territoire (valorisation des déchets, stimulation de filières économiques...).

Dans ce contexte, l'article présente les résultats d'une recherche effectuée dans l'agglomération de Port-au-Prince et dans une de ses communes, Tabarre, suite au séisme du 12 janvier 2010, afin d'analyser les dimensions spatiales de la gestion des déchets post-catastrophe. Cette analyse aide à comprendre la destination des $94 \%$ de 10 millions de $\mathrm{m}^{3}$ de déchets produits par le séisme qui n'ont pas été acheminés vers les centres officiels d'enfouissement et de valorisation. Elle permet en même temps d'appréhender les logiques spatiales de gestion des déchets mises en œuvre par les autorités nationales et internationales, comme par la société civile et le secteur informel, suite à la catastrophe. Il s'agit de logiques de relégation des déchets dans des espaces sacrifiés à cet égard. Cet éloignement s'opère à plusieurs échelles, tenant compte de l'utilisation du sol et de la proximité entre les gisements et les lieux d'évacuation des déchets. On retrouve alors une exacerbation des logiques déjà existantes pour les déchets ménagers et les déchets du bâtiment et des travaux publics de la ville.

Les constats opérés à Port-au-Prince vont alors dans le même sens que les retours d'expériences internationaux. Ces derniers préconisent dans la mesure du possible une gestion anticipée des déchets post-catastrophe, en particulier là où les catastrophes sont fréquentes, comme à Haïti, en aménageant notamment des lieux de dépôt final, ainsi que des espaces intermédiaires provisoires, permettant le transit et le tri des déchets.

\section{AUTHORS}

\section{ROXANA POPESCU}

Roxana Popescu, popescu.roxana.mihaela@gmail.com , est Géographe, Consultante en risques et vulnérabilité, ONG COOPI.

\section{MATHIEU DURAND}

Mathieu Durand, Mathieu.Durand@univ-lemans.fr, est Maître de Conférences en aménagement de l'espace et urbanisme à l'Université du Maine, chercheur au laboratoire ESO (UMR 6590 CNRS), responsable du Master 2 «Ingénierie des déchets ». Il a publié récemment :

- Durand M., 2012. Mesurer les inégalités environnementales et écologiques dans le contexte d'une ville en développement, les déchets et les eaux usées à Lima. Revue Flux, n 88/89

"Inégalités environnementales, services urbains et territoires », p. 67-78.

- Durand M., 2012. El sistema "compuesto" de manejo de residuos en Lima: como sacar provecho de las prácticas en las ciudades en desarrollo? Revue Perspectiva geográfica, Bogotá, UPTC, p. 35-47. - Durand M., 2011. La gestion des déchets et les inégalités environnementales et écologiques à Lima: entre vulnérabilité et durabilité. ESO travaux et documents, n³2, Rennes, UMR ESO 6590 CNRS, p. 15-23.

\section{ROBERT D'ERCOLE}

Robert D’Ercole, robert.dercole@ird.fr, est Directeur de recherche en géographie à l'IRD, UMR 8586 PRODIG.

- D'Ercole R., Hardy S., Metzger P., Robert J., Gluski-Chraibi P., 2012. Les dimensions spatiales et territoriales de la gestion de crise à Lima, Vertigo, vol. 12, $\mathrm{n}^{\circ}$ 1, [En ligne], http:// vertigo.revues.org/12009

- Metzger P., Robert J., Sierra A., D’Ercole R., Hardy S., Gluski P., 2013. Dimensions spatiales et territoriales de la gestion de crise : les ressources de décision et d'intervention à Lima et Callao. In Jost C. (dir.) Gestion du risque et sécurité civile : résilience, adaptation, stratégies. Revue Géographique 
de l'Est, 53 (1-2), p. 1-16. http://rge.revues.org/4576

- Robert J., D'Ercole R., 2014, à paraître. L'occupation et l'utilisation du sol en période de crise. Le cas de la commune de Tabarre (Port-au-Prince, Haïti) suite au séisme du 12 janvier 2010. Cybergeo (article relu et accepté). 